\title{
Reference values for hand grip strength in Japanese community-dwelling elderly: a meta-analysis
}

\author{
Naoto Kamide ${ }^{1,2} \cdot$ Ryo Kamiya $^{3} \cdot$ Tetsuharu Nakazono $^{4} \cdot$ Masataka Ando $^{5}$
}

Received: 28 May 2015/Accepted: 29 July 2015/Published online: 8 August 2015

(C) The Japanese Society for Hygiene 2015

\begin{abstract}
Objectives Reference values for hand grip strength in Japanese community-dwelling elderly independent in activities of daily living (ADLs) were calculated by metaanalysis.

Methods Papers reporting data on hand grip strength in elderly Japanese adults $\geq 60$ years of age and independent in ADLs were retrieved from electronic databases. Data were extracted from the selected papers and the weighted mean for hand grip strength by sex was calculated by random effect model. The association of hand grip strength with age and body weight was also analyzed using metaregression analysis.

Results Data for 15,784 individuals (5216 men and 10,568 women; mean age 67.0-79.8 years) were extracted from 97 sets of data from 33 papers. The weighted mean for hand grip strength was calculated as $33.11 \mathrm{~kg}$ [95\% CI 32.27-33.96] in men and $20.92 \mathrm{~kg}$ [95 \% CI 20.45-21.39] in women. A significant negative correlation was also seen between hand grip strength and age.
\end{abstract}

Naoto Kamide

naokami@kitasato-u.ac.jp

1 School of Allied Health Sciences, Kitasato University, 1-15-1 Kitasato, Minami-ku, Sagamihara, Kanagawa 252-0373, Japan

2 Graduate School of Medical Sciences, Kitasato University, Sagamihara 252-0373, Japan

3 Department of Rehabilitation, Seirei Hamamatsu City Rehabilitation Hospital, Hamamatsu 433-8511, Japan

4 Department of Rehabilitation, Kitasato University East Hospital, Sagamihara 252-0328, Japan

5 Department of Rehabilitation, Ushioda General Hospital, Yokohama 230-0001, Japan
Conclusions The mean hand grip strength of elderly people calculated in this study can be used as a reference value for the hand grip strength of Japanese communitydwelling elderly who are independent in ADLs. However, age needs to be considered in reference values for hand grip strength.

Keywords Japanese - Elderly · Hand grip strength · Reference values $\cdot$ Meta-analysis

\section{Introduction}

Hand grip strength measurements are simple and inexpensive, and are thus widely used as a means of evaluating muscle strength in the elderly. Hand grip strength is also used in elderly adults as a screening index for sarcopenia, which is defined as a decline in skeletal muscle mass and muscle function [1, 2]. Furthermore, poor hand grip strength is associated with mortality $[3,4]$ and disability $[4$, 5] in older adults and has been found to be a useful index for frailty [6, 7]. Reports have also emerged in recent years demonstrating a relationship between hand grip strength and oxidative DNA damage, which has been found to be related to cancer, heart disease, and diabetes [8]. Poor hand grip strength may, therefore, serve as a useful piece of information regarding the health of elderly people. However, appropriate reference values to serve as evaluation criteria are required to identify poor hand grip strength.

Several reports on reference values for hand grip strength exist among earlier studies $[9,10]$. However, other reports have suggested that racial differences exist in hand grip strength and other physical performance tests [11, 12]. Therefore, reference values not based on measurements in Asians [9] are not appropriate for Japanese people. In fact, 
different cut-off values are used for hand grip strength between Europe and Asia-even in screenings for sarcopenia [2]. Moreover, reference values for hand grip strength in Japanese people are based upon sample extractions from a limited geographical area, placing limitations on their generalization [10]. To set reference values for hand grip strength that can be adapted to Japanese people, data from a large sample obtained from a wide geographic area are needed. Thus far, we have used metaanalysis to integrate research data from various areas to more accurately set reference values for several motor function tests such as the Timed Up and Go test [12-15]. The aim of this study was to calculate reference values for hand grip strength in independent Japanese communitydwelling elderly using meta-analysis.

\section{Materials and methods}

\section{Paper retrieval method}

We examined peer-reviewed research papers reporting data on hand grip strength published from January 1983 to September 2014. The electronic databases used to retrieve the papers in question were PubMed, EMBASE, Cumulative Index to Nursing and Allied Health Literature (CINAHL), and Igaku Chuo Zasshi (an electronic database that consists of medical research articles in Japan). The search terms used were "hand grip strength/akuryoku," "hand strength," "grip," "grasp," "strength," "power," "older people/koureisya," "aged," and "elderly" in appropriate combinations. The selection criteria for papers used in the meta-analysis were set as follows in accordance with earlier studies [12-15]: (1) papers written in Japanese or English; (2) papers on Japanese community-dwelling people aged $\geq 60$ years old; (3) papers on community-dwelling elderly independent in activities of daily living (ADLs); (4) papers not examining frail elderly or elderly with an evident disease; (5) papers reporting measurements in kilograms or Newtons; (6) papers reporting data on hand grip strength by sex; and (7) papers listing the number of subjects and the mean and standard deviation (SD) of hand grip strength. Four researchers (NK, RK, TN, and MA) held discussions to determine search terms to use and which papers to include in the analysis.

Paper information (title, author(s), source), subject attributes (functioning, age, sex, body weight), measurement conditions, units of measurement, number of subjects, and the means and SDs of hand grip strength were extracted from papers that met the above selection criteria and were input into a database. If multiple data by sex and/ or age group were reported in a single paper, these were extracted as individual data points and input into the database.

\section{Statistical analysis}

To obtain the weighted mean of hand grip strength, the weighted mean and $95 \%$ confidence interval $(95 \% \mathrm{CI})$ were calculated from the mean and SD of each hand grip strength measurement. The $95 \%$ CI was calculated using the Knapp and Hartung method. To calculate the weighted mean, a DerSimonian-Laird random effect model was used. However, due to sex differences in hand grip strength [10], the weighted mean was analyzed separately for men and women. A meta-regression analysis was additionally used to analyze the association of hand grip strength with age and body weight. This meta-regression analysis was also conducted separately for men and women considering the effects of sex differences. For statistical processing, the statistical analysis software R Programming Language and Environment ( $\mathrm{R}$ version 3.1.3) [16] and the meta for package (version 1.9-4) [17] were used, with the level of statistical significance set at $5 \%$.

\section{Results}

The keyword search turned up 5340 papers. The titles and abstracts of the retrieved papers were checked and 4,819 papers that clearly did not meet the selection criteria were excluded. The main texts of the remaining 521 papers were checked, and a further 488 papers that did not meet the selection criteria were excluded. Thirty-three papers and 97 sets of data [5, 18-49] that met the selection criteria were ultimately subject to analysis (Fig. 1). The total number of subjects was 15,784 (5216 men and 10,568 women; mean age 67.0-79.8 years; Table 1). In 15 studies, measurements were taken in both upper limbs $(45.5 \%)$, with the highest value being reported. Measurements were taken in only the dominant upper limb in 10 studies (30.3\%). Sixteen studies $(48.5 \%)$ reported the measurement values of hand grip strength represented by the highest value, and 5 studies (15.2\%) reported those represented by the mean value. Detailed information of reported values (highest, mean, or median) was not described in 12 studies. Further, number of measurements of hand grip strength ranged from one to three times. With regard to measurement devices, a Smedley-type dynamometer was used in 29 studies (96.7 \%), whereas a Jamar-type dynamometer was used in only one study.

The weighted mean for hand grip strength calculated using the random effect model was $33.11 \mathrm{~kg}(95 \% \mathrm{CI}$ $32.27-33.96$ ) in men and $20.92 \mathrm{~kg}$ (95 \% CI 20.45-21.39) in women (Table 2). The effects of age and body weight 
Fig. 1 Selection flow of articles retrieved for meta-analysis

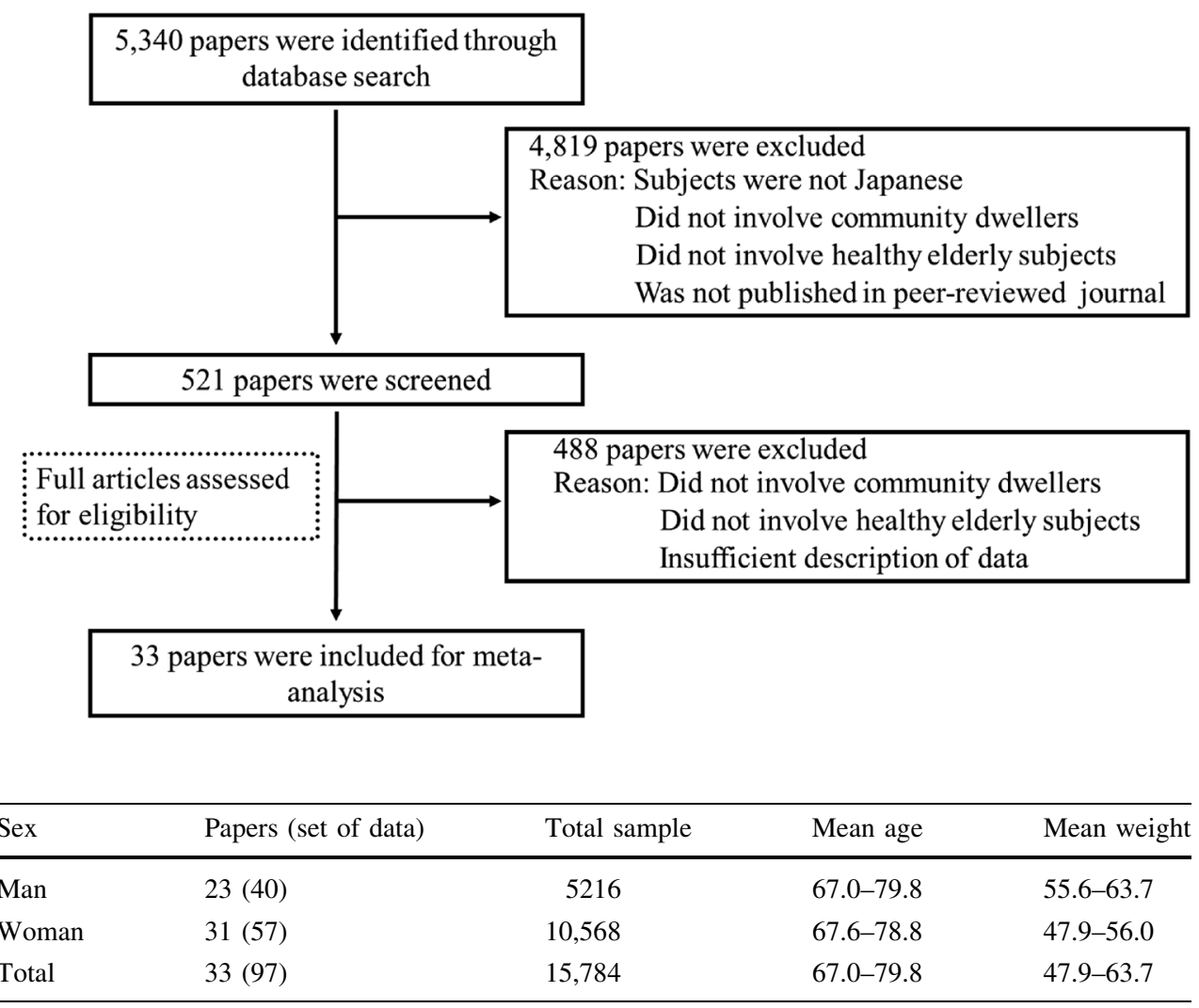

\begin{tabular}{llccc}
\hline Sex & Papers (set of data) & Total sample & Mean age & Mean weight \\
\hline Man & $23(40)$ & 5216 & $67.0-79.8$ & $55.6-63.7$ \\
Woman & $31(57)$ & 10,568 & $67.6-78.8$ & $47.9-56.0$ \\
Total & $33(97)$ & 15,784 & $67.0-79.8$ & $47.9-63.7$ \\
\hline
\end{tabular}

Table 1 Summary of samples and data

Table 2 Reference values for hand grip strength

\begin{tabular}{lll}
\hline Model & Sex & Weighted mean [95\% CI $]^{\mathrm{a}}(\mathrm{kg})$ \\
\hline Random effect $^{\mathrm{b}}$ & Man & $33.11[32.27: 33.96]$ \\
& Woman & $20.92[20.45: 21.39]$ \\
Mixed effect $^{\mathrm{b}, \mathrm{c}}$ (meta-regression) & Man & $=78.74-0.62[-0.51:-074]^{*}$ Age $[F(3,32)=51.33, p<0.0001]$ \\
& Woman & $=46.90-0.35[-0.21:-0.43]^{*}$ Age $[F(1,48)=24.01, p<0.0001]$
\end{tabular}

a $95 \%$ CIs were estimated using the Knapp and Hartung adjustment

b DerSimonian-Laird estimator was used for estimation of models

c Six data for men and 7 for women were excluded from the Mixed effect model because they lacked data on age

were then analyzed by meta-regression analysis. Results did not reveal a statistically significant association between hand grip strength and body weight in men or women (men, regression coefficient $=0.42, P=0.30$; women, regression coefficient $=0.22, P=0.20$ ). On the other hand, a significant negative correlation was seen between age and hand grip strength (Fig. 2). The age-adjusted weighted mean was demonstrated using the formula in Table 2. This formula shows that hand grip strength decreased by a mean $3.10 \mathrm{~kg}$ and $1.75 \mathrm{~kg}$ every 5 years in men and women, respectively. Furthermore, this formula was used to calculate the mean hand grip strength in men aged 65,75 , and 85 years as $38.44,32.24$, and $26.04 \mathrm{~kg}$, respectively, whereas the mean in women aged 65,75 , and
85 was calculated as $24.15,20.65$, and $17.15 \mathrm{~kg}$, respectively.

\section{Discussion}

In this study, we limited subjects to a specific subset of participants, i.e., Japanese community-dwelling elderly who were independent in ADLs, to accurately calculate reference values for hand grip strength by meta-analysis. The data used in this analysis were taken from samples of participants from different geographic areas, and the total number of samples was large (approximately 5000 men and 10,000 women). In addition, an obvious publication 

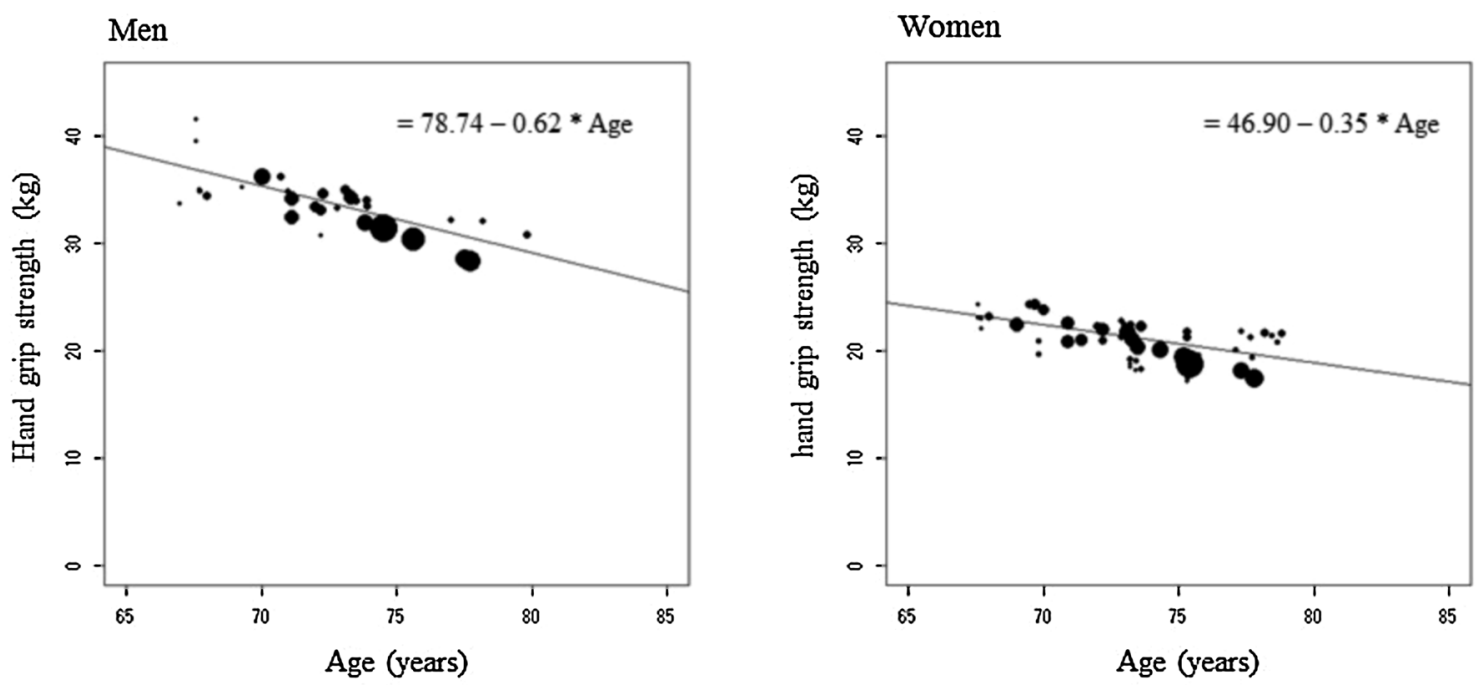

Fig. 2 Scatter plots of age versus hand grip strength based on meta-regression analysis

bias, judged from a funnel plot (plot not shown), was not found in this study. Therefore, the results of this study enabled us to present reference values for hand grip strength in elderly Japanese adults leading independent lives in the community. Notably, more than $70 \%$ of the data analyzed in this study reflected measurements taken in the dominant hand, where the highest value for the left or right hand was recorded. Hand grip strength is reportedly greater in the dominant hand than the non-dominant hand $[21,49]$. The reference values in this study may, therefore, reflect the highest value for individual hand grip strength. Moreover, the Smedley-type dynamometer was used for measurements of hand grip strength in almost all included studies. Accordingly, the reference values in this study reflect values for the Smedley-type dynamometer.

As for racial differences, Asians are considered to have poorer hand grip strength than Caucasians [2]. A study in Italians found that the mean hand grip strength of people aged $65-74$ years was $39.2 \mathrm{~kg}$ in men and $22.2 \mathrm{~kg}$ in women [50]. When mean hand grip strength in elderly Japanese adults aged $65-74$ years is calculated using the formula in Table 2, the results are $35.7 \mathrm{~kg}$ in men and $22.6 \mathrm{~kg}$ in women. Hand grip strength, therefore, tends to be poorer in Japanese men when compared to Italian men. On the other hand, there is no marked difference in strength between elderly Japanese women versus elderly Italian women. A study comparing hand grip strength between elderly native Japanese women and Caucasian women found that hand grip strength was greater in native Japanese women than in Caucasian women [11]. It is, therefore, likely that racial differences in hand grip strength may also differ between the sexes. It is thus important to set reference values taking racial differences into account. It has also been reported that racial differences exist among
Asians in terms of respiratory muscle strength [51]. We cannot completely rule out the possibility that, like the respiratory muscles, there are differences between Asian geographic areas in hand grip strength. It is thus necessary to set reference values for determining the state of hand grip strength in elderly according to each individual race. However, further investigation and analysis may be necessary to clarify the racial difference in hand grip strength, since it cannot be analyzed from just the results of this study.

This study demonstrates that hand grip strength declines with age in both men and women. This age-related decline in hand grip strength has been similarly reported in earlier studies $[6,10,50]$. These results are, therefore, not surprising. Reference values need to take into account the effects of age when the state of hand grip strength in elderly people is being determined. On the other hand, no association was seen between hand grip strength and body weight. A study that examined young people also found no clear association between hand grip strength and physique [52]. Body weight is generally known to have a large effect on muscle strength in the lower limbs; however, findings have suggested that the effect of body weight on hand grip strength is small. The need to take into account body weight in reference values for hand grip strength can be considered minor. Further, some degree of heterogeneity was found in the measurement method of hand grip strength (i.e., number of measurements, representation of measurement value). Generally, the influence of this heterogeneity is thought to be small because it is well known that measurements of hand grip strength are generally reliable. However, the influence of heterogeneity in the measurement method should still be noted as a limitation of this study. 
In conclusion, reference values for hand grip strength in Japanese community-dwelling elderly leading independent lives were demonstrated by meta-analysis in this study. Hand grip strength is a simple motor function test that can provide information about the health of elderly people. The reference values in this study can be used to easily determine the state of hand grip strength in Japanese community-dwelling elderly. However, in general, it is important to take into account the age of subjects when determining the reference values for hand grip strength in specific subsets of a population.

Acknowledgments This study was partially funded by the Ministry of Education, Culture, Sports, Science and Technology (Grant-in-Aid for Young Scientists (B) No. 25870703). The funder had no role in study design, data collection and analysis, or the decision to publish.

\section{Compliance with ethical standards}

Conflict of interest There are no conflicts of interest regarding the content of this study.

\section{References}

1. Cruz-Jentoft AJ, Baeyens JP, Bauer JM, Boirie Y, Cederholm T, Landi F, et al. Sarcopenia: European consensus on definition and diagnosis: report of the European working group on sarcopenia in older people. Age Ageing. 2010;39:412-23.

2. Chen L-K, Liu L-K, Woo J, Assantachai P, Auyeung T-W, Bahyah KS, et al. Sarcopenia in Asia: consensus report of the Asian working group for sarcopenia. J Am Med Dir Assoc. 2014;15:95-101.

3. Sasaki H, Kasagi F, Yamada M, Fujita S. Grip strength predicts cause-specific mortality in middle-aged and elderly persons. Am J Med. 2007;120:337-42.

4. Bohannon RW. Dynamometer measurements of hand-grip strength predict multiple outcomes. Percep Motor Skills. 2001;93:323-8.

5. Akune T, Muraki S, Oka H, Tanaka S, Kawaguchi H, et al. Incidence of certified need of care in the long-term care insurance system and its risk factors in the elderly of Japanese populationbased cohorts: The ROAD study. Geriatr Gerontol Int. 2014;14:695-701.

6. Syddall H, Cooper C, Martin F, Briggs R, Sayer AA. Is grip strength a useful single marker of frailty? Age Ageing. 2003;32:650-6.

7. Fried LP, Tangen CM, Walston J, Newman AB, Hirsch C, Gottdiener J, et al. Frailty in older adults: evidence for a phenotype. J Gerontol Med Sci. 2001;56A:M146-56.

8. Muzembo BA, Nagano Y, Eitoku M, Ngatu NR, Matsui T, Bhatti SA, et al. A cross-sectional assessment of oxidative DNA damage and muscle strength among elderly people living in the community. Environ Health Prev Med. 2014;19:21-9.

9. Bohannon RW, Bear-Lehman J, Desrosiers J, Massy-Westropp $\mathrm{N}$, Mathiowetz V. Average grip strength: a meta-analysis of data obtained with a jamar dynamometer from individuals 75 years or more of age. J Geriatr Phys Ther. 2007;30:28-30.

10. Yoshimura $\mathrm{N}$, Oka $\mathrm{H}$, Muraki S, Akune $\mathrm{T}$, Hirabayashi $\mathrm{N}$, Matsuda S, et al. Reference values for hand grip strength, muscle mass, walking time, and one-leg standing time as indices for locomotive syndrome and associated disability: the second survey of the ROAD study. J Orthop Sci. 2011;16:768-77.
11. Aoyagi K, Ross PD, Nevitt MC, Davis JW, Wasnich RD, Hayashi $\mathrm{T}$, et al. Comparison of performance-based measures among native Japanese, Japanese-Americans in Hawaii and Caucasian women in the United States, ages 65 years and over: a crosssectional study. BMC Geriatr. 2001;1:3.

12. Kamide N, Takahashi K, Shiba Y. Reference values for the Timed Up and Go test in healthy Japanese elderly people: determination using the methodology of meta-analysis. Geriatr Gerontol Int. 2011;11:445-51.

13. Kamide N, Takahashi-Narita K, Kawamura A, Mizuno K, Shiba Y. Determination of the reference value and systematic bias of the functional reach test in Japanese elderly people by metaanalysis. J Clin Gerontol Geriatr. 2012;3:122-6.

14. Ando M, Kamide N. The reference values for the $5 \mathrm{~m}$ walking time in community-dwelling Japanese elderly people: determination using the methodology meta-analysis. Sogo Rihabiriteshon. 2013;41:961-7 (in Japanese).

15. Nakazono T, Kamide N, Ando M. The reference values for the chair stand test in healthy Japanese older people: determination by meta-analysis. J Phys Ther Sci. 2014;26:1729-31.

16. $\mathrm{R}$ core Team. R: A language and environment for statistical computing, $\mathrm{R}$ foundation for statistical computing. Vienna. (2015). http://www.R-project.org/. Acccessed 9 Mar 2015.

17. Viechtbauer $\mathrm{W}$. Conducting meta-analyses in $\mathrm{R}$ with the metafor package. J Stat Softw. 2010;36:1-48.

18. Aoyagi Y, Park H, Watanabe E, Park S, Shephard RJ. Habitual physical activity and health-related quality of life in older adults: interactions between the amount and intensity of activity (the Nakanojo Study). Gerontology. 2009;55:523-31.

19. Ishizaki T, Furuna T, Yoshida Y, Iwasa H, Shimada H, Yoshida $\mathrm{H}$, et al. Declines in physical performance by sex and age among nondisabled community-dwelling older Japanese during a 6 year period. J Epidemiol. 2011;21:176-83.

20. Kanagae M, Abe Y, Honda S, Takamura N, Kusano Y, Takemoto $\mathrm{T}$, et al. Determinants of self-rated health among communitydwelling women aged 40 years and over in Japan. Tohoku J Exp Med. 2006;210:11-9.

21. Kubota H, Demura S, Kawabata H. Laterality and age-level differences between young women and elderly women in controlled force exertion (CFE). Arch Gerontol Geriatr. 2012;54:e68-72.

22. Kwon J, Suzuki T, Yoshida H, Kim H, Yoshida Y, Iwasa H. Concomitant lower serum albumin and vitamin D levels are associated with decreased objective physical performance among Japanese community-dwelling elderly. Gerontology. 2007:53:322-8.

23. Morita M, Takamura N, Kusano Y, Abe Y, Moji K, Takemoto T, et al. Relationship between falls and physical performance measures among community-dwelling elderly women in Japan. Aging Clin Exp Res. 2005;17:211-6.

24. Nakagawa K, Inomata N, Nakazawa R, Sakamoto M. The effect of a health promotion program consisting of easy and simple exercises for community living elderly people. J Phys Ther Sci. 2007:19:235-42.

25. Nakamura $K$, Saito $T$, Nishiwaki $T$, Ueno $K$, Nashimoto $M$, Okuda Y, et al. Correlations between bone mineral density and demographic, lifestyle, and biochemical variables in communitydwelling Japanese women 69 years of age and over. Osteoporos Int. 2006;17:1202-7.

26. Nakamura K, Oyama M, Saito T, Oshiki R, Kobayashi R, Nishiwaki $T$, et al. Nutritional and biochemical parameters associated with 6 year change in bone mineral density in community-dwelling Japanese women aged 69 years and older: The Muramatsu Study. Nutrition. 2012;28:357-61.

27. Nakao H, Yoshikawa T, Mimura T, Hara T, Nishimoto K, Fujimoto $\mathrm{S}$. Influence of lower-extremity muscle force, muscle mass 
and asymmetry in knee extension force on gait ability in community-dwelling elderly women. J Phys Ther Sci. 2006;18:73-9.

28. Sugiura Y, Tanimoto Y, Watanabe M, Tsuda Y, Kimura M, Kusabiraki T, et al. Handgrip strength as a predictor of higherlevel competence decline among community-dwelling Japanese elderly in an urban area during a 4 year follow-up. Arch Gerontol Geriatr. 2013;57:319-24.

29. Suzuki T, Kim H, Yoshida H, Ishizaki T. Randomized controlled trial of exercise intervention for the prevention of falls in community-dwelling elderly Japanese women. J Bone Miner Metab. 2004;22:602-11.

30. Suzuki T, Kwon J, Kim H, Shimada H, Yoshida Y, Iwasa H, et al. Low serum 25-hydroxyvitamin D levels associated with falls among Japanese community-dwelling elderly. J Bone Miner Res. 2008;23:1309-17.

31. Tanaka K, Kim HS, Yang JH, Shimamoto H, Kokudo S, Nishijima T. Index of assessing functional status in elderly Japanese men. Appl Human Sci. 1995;14:65-71.

32. Tanimoto Y, Watanabe M, Sun W, Tanimoto K, Shishikura K, Sugiura $\mathrm{Y}$, et al. Association of sarcopenia with functional decline in community-dwelling elderly subjects in Japan. Geriatr Gerontol Int. 2013;13:958-63.

33. Yoshihara A, Tobina T, Yamaga T, Ayabe M, Yoshitake Y, Kimura $\mathrm{Y}$, et al. Physical function is weakly associated with angiotensin-converting enzyme gene $\mathrm{I} / \mathrm{D}$ polymorphism in elderly Japanese subjects. Gerontology. 2009;55:387-92.

34. Yoshimatsu T, Yoshida D, Shimada H, Komatsu T, Harada A, Suzuki T. Relationship between near-infrared spectroscopy, and subcutaneous fat and muscle thickness measured by ultrasonography in Japanese community-dwelling elderly. Geriatr Gerontol Int. 2013;13:351-7.

35. Inagaki A, Sakurai R, Hirano W, Takanami R, Mizoguchi W, Iwasaki Y, et al. Effectiveness of care prevention exercise "Ogenki Shan-Shan Taisou". Jpn J Nurs Health Sci. 2012;10:47-56 (in Japanese).

36. Okazaki D, Kohda M, Miyaguchi H, Kawamura H, Tsurumi M, Tsujishita M. Effect of gender difference on gait and physical fitness level in healthy elderly people.-Influence of visual feedback. J Hiroshima Phys Ther. 2002;11:20-5 (in Japanese).

37. Kono R. Relationship between occlusal force and preventive factors for disability among community-dwelling elderly persons. Jpn J Geriatr. 2009;46:55-62 (in Japanese).

38. Iwase H, Murata S, Miyazaki J, Otao H, Horie J. Factors influencing the presence or absence of getting down on all fours on standing up from the floor. Jpn J Health Promotion Phys Therepy. 2012;2:101-6 (in Japanese).

39. Miyabara Y, Takeshita J. The Relation of exercise performance with healthy life span in the community dwelling elderly-a 3 year follow-up study. J Jpn Phys Therapy Assoc. 2004;31:155-9 (in Japanese).

40. Gushiken S, Oi N, Tobimatsu Y, Iwaya T. The relevance of standing posture and walking function in elderly people. J Phys Med. 2003;14:241-8 (in Japanese).
41. Goto R, Higashi M, Nagai Y, Yoshida S, Goto S, Hunahashi H, et al. Relationship between age-related changes on physical function and physical activity in community-dwelling older adults. J Aichi Soc Phys Therapy. 2012;24:60-5 (in Japanese).

42. Hirota C, Watanabe M, Tanimoto Y, kono R, Higuchi Y, Kono K. A cross-sectional study on the relationship between the trail making test and mobility-related functions in community-dwelling elderly. Jpn J Geriatrics. 2008;45:647-54 (in Japanese).

43. Edo A, Murata S, Horie J, Otao H, Murata J, Miyazaki J, Yamasaki S, et al. The significance of assessing finger motor function in community dwelling elderly people-comparison between old female and old male. Jpn J Health Prom Phys Therepy. 2012;2:47-52 (in Japanese).

44. Asakawa Y, Endo F, Yamaguchi Y, Takahashi R. The characteristics of participants and the effects of self-paced resistance training for community-dwelling elders in early period of a residents-led preventive care program, the Onishi model. J Jpn Phys Therapy Assoc. 2008;35:229-36 (in Japanese).

45. Murata S, Kai Y, Mizota K, Yamasaki S, Yumioka M, Otao H, et al. Relationship between one-leg standing duration with vision and physical function among community dwelling older adults. Rigakuryoho Kagaku. 2006;21:437-40 (in Japanese).

46. Nakamura I, Okuda M, Kage H, Kunitsugiu I, Sugiyama S, Hobara T, et al. The relationship between a functional reach test and other balance tests. Rigakuryoho Kagaku. 2006;21:335-9 (in Japanese).

47. Fujiwara N, Sato J, Sakuma S, Katsuse S, Araki A, Fukuharu M, et al. Association between oral condition with tooth brushing frequency and physical strength in free-living elderly people. Bull Soc Med. 1999;17:29-37 (in Japanese).

48. Michishita R, Fukae C, Mihara R, Ikenaga M, Morimura K, Takeda N, et al. Association between physical performance and electrocardiographic heart rate corrected-QT interval in elderly subjects. Jpn J Phys Fit Sport Med. 2013;62:283-91 (in Japanese).

49. Hatta M, Otomo E, Yoshida H, Takaira Y, Ohtake N. Grip, pinch strength and motor hand skill in the elderly: relationship to age, sex and anthropometric factors. Sogo Riha. 1993;21:489-92 (in Japanese).

50. Lauretani F, Russo CR, Bandinelli S, Bartali B, Cavazzini C, Di Iorio A, et al. Age associated changes in skeletal muscles and their effect on mobility: an operational diagnosis of sarcopenia. J Appl Physiol. 2003;95:1851-60.

51. Johan A, Chan CC, Chia HP, Chan OY, Wang YT. Maximal respiratory pressures in adult Chinese. Malays Indians. Eur Respir J. 1997;10:2825-8.

52. Lad UP, Satyanarayana P, Shisode-Lad S, Siri CC, Kumari NR. A study on the correlation between the body mass index (BMI), the body fat percentage, the handgrip strength and the handgrip endurance in underweight, normal weight and overweight adolescents. J Clin Diagn Res. 2013;7:51-4. 